\title{
The optimization model for designing parameters of creative folding table
}

\author{
Jia Liu \\ Department of Electrical Engineering, North China Electric Power University, Baoding, China
}

1277565033@qq.com

Keywords: flat folding table, projection, trigonometric function, optimization model.

\begin{abstract}
The author of this paper solves the problem about the designing parameters of folding table, comprehensively use projection method, the numerical calculation, physical analysis and solution of triangle method, and establish the optimization model for the design of the processing parameters. Simplify the table structure into line segments, and then get the wood structure of triangle relation by the projection method. Last solve the triangle by the cosine theorem; According to the principle of stability and degree of processing, minimizing the total length of slots as the optimization target, reinforced location as constraint conditions, and then, appropriate optimization model is established, the problem of folding tables for designing process parameters is solved
\end{abstract}

\section{Introduction}

Desktop of creative folding table assumes the circular, table legs as the hinge of the activity can be split into a flat, it's convenient and beautiful. But so far the study of folding table is how to effectively use or save space ${ }^{[1]}$, others from the perspective of industrial design, the three aspects of the structure, material, function of the folded product features are summarized ${ }^{[2]}$, but the lack of guidance of folding table parameters in the design. Others about the design of the folding table parameters abstraction table into simple mathematical structure, ignoring the actual production materials to the design requirements. For example in literature 3, the unconstrained optimization model is established to solve the parameter, without considering the thickness of the table legs as the effects of designing parameters of flat folding table.

This paper folding table design requirements of good stability, material minimum principle, and considering the actual situation and the universality of the model, for a given height and diameter round table, folding table to slot with target of minimizing the total length, reinforced position as constraint conditions, the optimization model is established, rectangular plate materials are presented and folding table of the mathematical model of optimal design of the processing parameters and procedures. This has more practical reference value.

\section{The Assumption of Model}

1. There is no gap between table legs, the sum of all the table leg width is the width of the desktop.

2. Table material used for wood, the specification is consistent, uniform thickness and the same.

3. Table pressure within the scope of legs compressive ability, regardless of the legs bent wood.

\section{Optimization model for the design of the processing parameters}

Base on the slenderness ratio of wood width ${ }^{[4]}$. Slenderness ratio refers to the calculation of bar length and the ratio of the radius of gyration of bar section. The slenderness ratio shall be calculated on the formula $\lambda=\left(\sqrt{12} l_{0}\right) / \beta$. We can see from the formula that slenderness ratio of bar with only calculate the length and the width of section. According to the GBJ 5- $73^{[5]}$ specification for design of timber structure selection standard, the slenderness ratio of compressive bar should comply with the regulations of the state, rods of the same material slenderness ratio is the same. 
Because the outermost wood is the main bearing structure, as long as the outermost wood slenderness ratio within the rules. Known lateral batten is $52.19 \mathrm{~cm}$ long, $2.5 \mathrm{~cm}$ wide, the actual length should be multiplied by the coefficient of 0.8 for the calculation of batten length, and plug type to get slenderness ratio bars made of this kind of plate material is 57.85 . So make sure the outside piece, batten length can get wood width $\beta=(\sqrt{12} \times 0.8 l) / 57.85$.

The angle between the outermost table leg and desktop. When considering the design's stability, first of all should make sure position of the outermost table legs. This article will remove auxiliary reinforced structure, mechanical properties ensuring table form the basic structure of the framework is shown in figure 1, the picture (a) is the main view, the graph (b) is top view, the circular projection area to the desktop, consisting of rectangular area as the lateral leg area. Literature [5], the legs in the vertical direction projection of a rectangular area including desktop area, the table structure, steadily when legs cut inside and desktop projection of the region composition, legs and desktop Angle is the largest, desktop diameter and the height of the table must have, at this time the lateral length of the shortest batten, supplies at least.

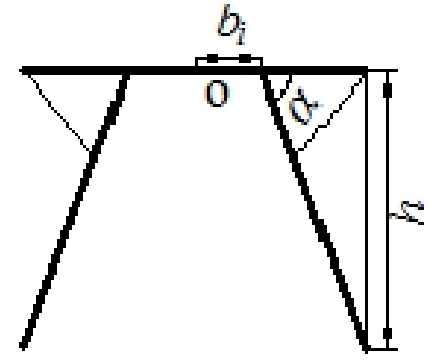

(a)front view

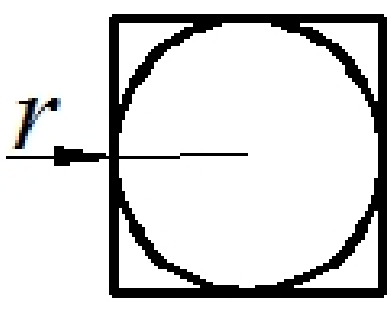

(b) top view

Fig. 1 Stress the basic structure of the table view

At the lateral batten hinge point to the center distance of desktop is $b_{n}=\sqrt{40^{2}-(40-0.5 \beta)^{2}}$, set the desktop height is $h$, desktop diameter $D$, table still is $3 \mathrm{~cm}$ thick, the most lateral batten with the Angle of the desktop is $\alpha=\arctan \left[(h-3) /\left(0.5 \mathrm{D}-b_{n}\right)\right]$. For all kinds of the legs of the table, the above rules are set up, so most of the lateral batten length is: $l=(h-3) / \sin \alpha$.

\section{The optimization model of design processing parameter of creative flat folded into a table.}

The tablet size. Set the desktop height is $h$, desktop diameter $D$, wood width for $\beta$, each side of the table to the total number of wood for $N=2 n=80 / \beta$ (root). According to the formula $b_{i}=\sqrt{r^{2}-[0.5 \beta+\beta(i-1)]^{2}}$ to calculate the lateral batten and desktop vertical distance to center of the circle $b_{n}, r$ is the radius of the desktop. The lateral batten reasonable length is known to $l=(\mathrm{h}-3) / \sin \alpha$, so machining the long rectangular plate of the table for $2\left(b_{n}+l\right)$, the size of the plate material to $2\left(b_{n}+l\right) \mathrm{cm} \times D \mathrm{~cm} \times 3 \mathrm{~cm}$.

The determination of reinforced location. The model's optimization goal. Set $p$ as distance from the outermost wood to reinforced the position of the hinge at the desktop, $d_{i}$ is slotted length by wood $i, W$ for the quantity of slot. Slot thickness to a certain value of $\lambda$, the $\lambda$ is based on a fixed value, so in the optimization model can not consider $\lambda$, and directly to the slot length is the sum of the minimum as the optimization goal. Therefore, to establish the optimization goal is $\min W=\sum_{i=1}^{n} d_{i}$. According to the figure 2 , calculate slot length in the following formula.

$$
\left\{\begin{array}{l}
a_{i}=b_{i}-b_{n} \\
b_{i}=\sqrt{r^{2}-[0.5 \beta+\beta(i-1)]^{2}} \\
c_{i}=\sqrt{a_{i}^{2}+p^{2}-2 p a_{i} \cos \alpha} \\
d_{i}=c_{i}-\left(p-a_{i}\right)
\end{array}\right.
$$




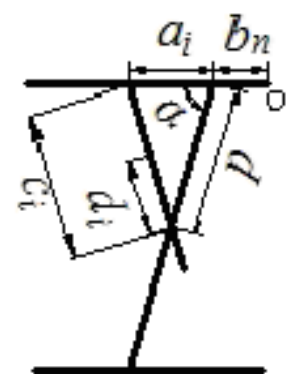

Fig. 2 Table leg structure projection drawing

The model's constraints. Observe batten in longitudinal and cross section can be seen that the direction of the projection reinforced location can not be infinite close to the outside piece, otherwise some wood in the inside of the slot will beyond the end of the wood, not fixed. Therefore the maximum length of the $p$ is limited by the medial batten length. So when the ends of the innermost wood connected with reinforced obtained, get the maximum value of $p$. When

$$
c_{1}=\sqrt{a_{1}^{2}+p^{2}-2 p a_{1} \cos \alpha}=l-a_{1} \text {, solve } p_{\max } \text {. }
$$

By the same token, reinforced location in a shortest batten with desktop hinge, reinforced position at this time to the lateral batten hinge is equal to the longest and the shortest distance difference in the length of sticks, namely $p_{\min }=a_{1}$. So the reinforced location as constraint conditions is $p_{\min }<p<p_{\max }$.

Reinforced location optimization design model. Considering the above objective function and constraint conditions, then we can get optimal design for reinforced location planning model.

$$
\begin{aligned}
& \min W=\sum_{i=1}^{n} d_{i} \\
& \left\{\begin{array}{l}
a_{i}=b_{i}-b_{n} \\
b_{i}=\sqrt{r^{2}-[0.5 \beta+\beta(i-1)]^{2}} \\
c_{i}=\sqrt{a_{i}^{2}+p^{2}-2 p a_{i} \cos \alpha} \\
d_{i}=c_{i}-\left(p-a_{i}\right)
\end{array}\right. \\
& \text { s.t. } p_{\min }<p<p_{\max }
\end{aligned}
$$

Using MATLAB ${ }^{[6]}$ to solve the model (2), get the total slot length as a function of reinforced location diagram is shown in figure 3 . According to the regulation ${ }^{[5]}$, reinforced position to the shortest distance of the end of a piece of wood should not be less than $8.4 \mathrm{~cm}$, from figure $3 \mathrm{know}, W$ and $p$ to monotone decreasing relationship, so when the reinforced location to the end of the shortest wood is equal to $8.4 \mathrm{~cm}$, the largest $p, W$ minimum objective function of optimal results.

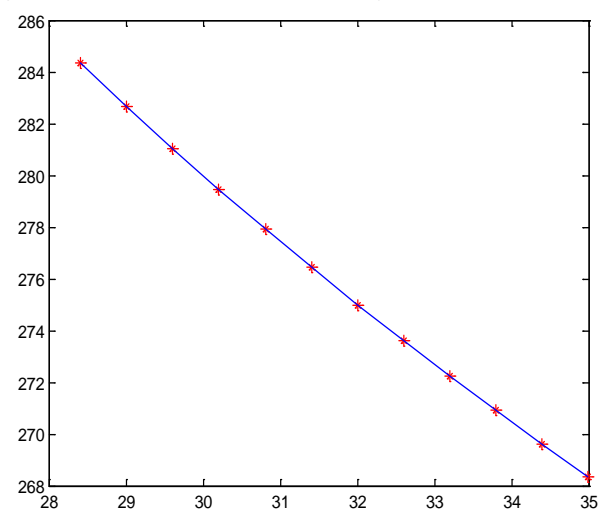

Fig.3 W and p diagram

The optimum design of folding table processing parameters solving process. To sum up, given the height of the table $h$ and circular diameter $D$, desktop can work out the optimum design of plate materials and folding table processing parameters, solving process as shown in figure 4 . 


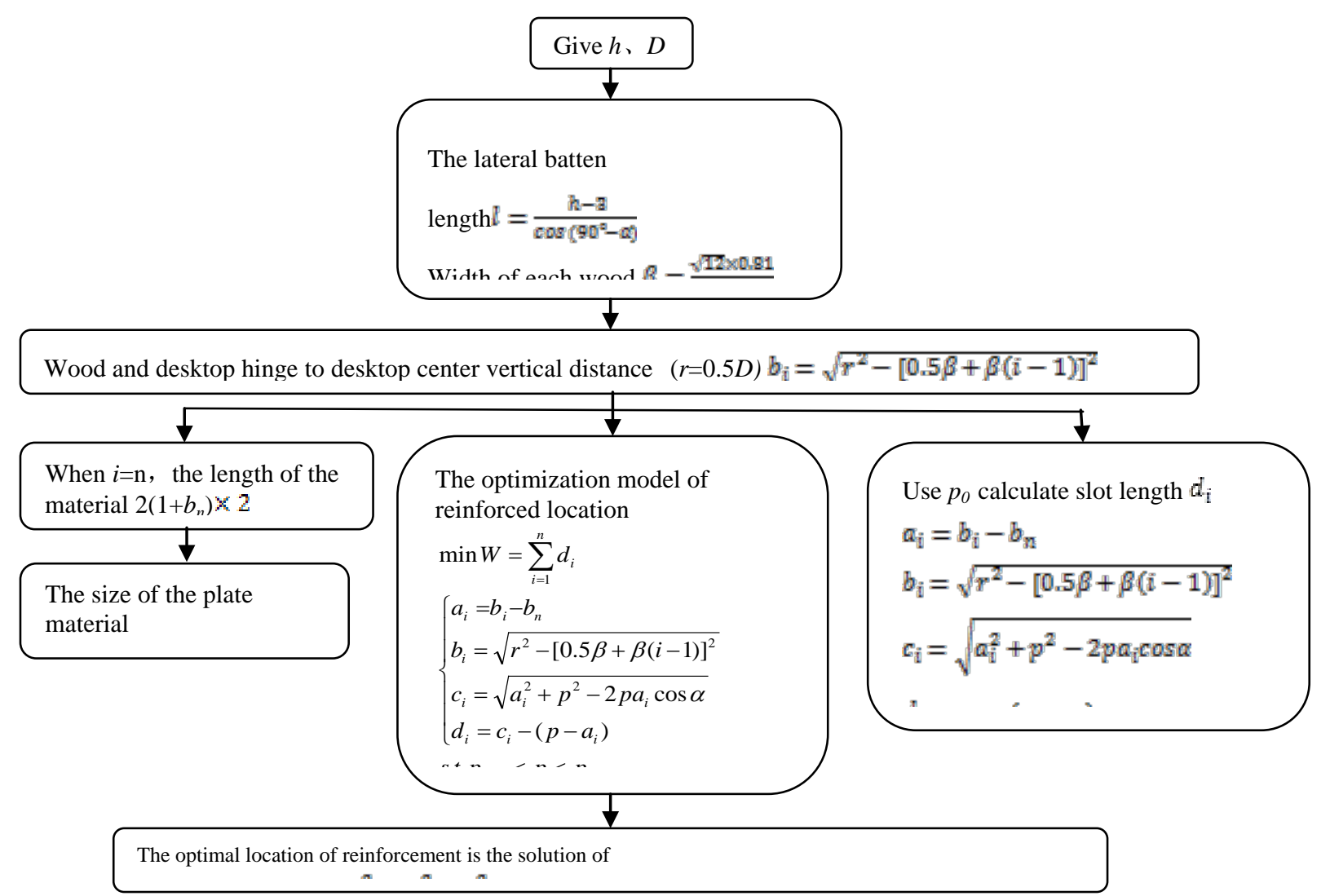

Fig.4 For the optimum design of plate and folding table processing parameters of flow chart

\section{Conclusions}

This article through to simplify the table structure into several segments, projection method is used to get the wood structure of triangular relationship, facilitate mathematical analysis and calculation, got the optimal design of the table processing parameters. In optimization model, the definition of slenderness ratio are batten in proportion to the width and length of relationship, conform to the requirement of practical production, the results of the model is accurate and reliable.

This paper mostly uses the basic knowledge and understanding of geometric calculation and knocking down to resolve the problem, thinking is concise, the method is effective. And reduction on the model by mathematics, based on the analysis of the stability of the structure of desktop table design parameter of the optimal solution, easy to analyze the rationality of the table under different design conditions, this method can be applied to other structure design of furniture.

\section{References}

[1] Jiaxin Lin, Guiping Nie. Fold furniture design based on TRIZ theory study [J]. Journal of Donghua university (natural science edition), 2011, 04:518-521.

[2] Xiaotong Jin. Theory of the folding furniture and its application in modern design [D]. Kunming University of science and technology, 2011.

[3] Kai An, Hongbing Yu, Xiaoyu Zhang, Yinfeng Wang. Creative folding table design based on unconstrained optimization [J]. Journal of Fujian computer, 2014 preceding: 24 to 25.

[4] Shoukui Si, Xiqing Sun. Mathematical modeling algorithm and application [M]. Beijing: national defence industry press, 2014.2.

[5] GB50005 specification for design of timber structure [S].

[6] Jinwu Zhuo. Application of MATLAB in mathematical modeling [M]. Beijing: Beijing University of aeronautics and astronautics press, 2011.4. 